\title{
Les constructions résultatives des verbes de changement chromatique en français
}

\author{
Resultative Constructions and Verbs of Chromatic Change in French
}

KURZFASSUNG: Dieser Beitrag präsentiert eine korpuslinguistische Untersuchung von Konstruktionen, die mit Hilfe der Verben peindre und colorer einen Farbwechsel ausdrücken. In einer als verb-framed geltenden Sprache wie dem Französischen stellen derartige resultative Konstruktionen eine typologische Anomalie dar. Unsere Studie zeigt drei Aspekte auf: (i) Entgegen den allgemeinen Annahmen toleriert das Französische in geringem Maße und unter spezifischen Bedingungen adjektivische resultative Ergänzungen; (ii) die Bandbreite der möglichen Präpositionen ist größer als allgemein angenommen, wobei instrumentale Konstruktionen (mit à, par, avec) von ,echten' Resultativkonstruktionen (mit en, de) zu unterscheiden sind; (iii) die Wahl zwischen de und en ist nicht zufällig. Sie orientiert sich an den diathetischen Eigenschaften des Prädikats und an der ,Betroffenheit' der Prädikationsbasis. Letztlich lassen sich die verschiedenen Konstruktionen auf einer Skala anordnen, die die Nähe zu der prototypischen Resultativkonstruktion angibt.

Schlagworte: Resultativität, Farbwechsel, Verb, Präposition, verb-framed, Typologie

ABSTRACT: This paper proposes a corpus-based analysis of a set of constructions expressing chromatic change with peindre and colorer as 'emblematic' verbs. In a verb-framed language such as French, such resultative constructions constitute a typological anomaly. This paper presents three advances: (i) contrary to the common view, French does tolerate adjectival resultative complements, albeit marginally and under specific conditions; (ii) the array of prepositions found in these constructions is larger than commonly accepted, although one has to distinguish instrumental $(\dot{a}, p a r, a v e c)$ constructions and resultative constructions proper (en, $d e)$; (iii) the choice between de and $e n$ is not arbitrary, but oriented by the diathetic properties of the predicate and by the affectedness of the predication base. The constructions can be put on a scale of resultativeness.

Keywords: resultative, chromatic change, verb, preposition, verb-framed, typology

1 Nous tenons à remercier Justine Métairy pour avoir diffusé le questionnaire parmi les locuteurs natifs. Un tout grand merci aussi à Marleen Van Peteghem pour ses remarques à propos d'une version antérieure de ce texte. Nous remercions aussi les lecteurs de la ZFSL pour la relecture critique. 


\section{Introduction : les verbes de changement chromatique au sein des constructions résultatives}

Les verbes $(\mathrm{V})$ de changement chromatique constituent une classe de verbes intéressante dans la mesure où ils prennent un complément accessoire (un SX, c'est-à-dire un constituant à caractère morphosyntaxique variable) qui identifie le résultat d'un changement d'état, en l'occurrence chromatique, comme le montre l'implication suivante :

(1) Il a peint la porte en vert $\rightarrow$ la porte est verte maintenant ; elle a changé de couleur.

De ce fait, ces constructions appartiennent à la famille des constructions résultatives (analytiques ; ${ }^{2}$ e. a. Boas 2003), dont il convient de rappeler ici l'exemple emblématique :

(2) John hammered the metal flat. (Wechsler 2001 ; cf. aussi Boas 2011)

Les constructions résultatives présentent un intérêt particulier pour la typologie des langues. En effet, comme le résultat d'un changement (en l'occurrence chromatique) peut être considéré comme une métaphore de la destination d'un déplacement (angl. goal) orienté dans l'espace, ${ }^{3}$ les constructions résultatives ont pu être appréhendées à travers une opposition typologique élaborée pour l'analyse des verbes de mouvement : verb-framed vs satellite-framed (e. a. Talmy 1991, 2000 ; Slobin 2004). Les langues satelliteframed expriment - prototypiquement - la destination du mouvement par un constituant périphérique (ou par une particule ou un affixe : p. ex. néerl. weggaan 'partir'), alors que les langues verb-framed l'incorporent dans le radical du verbe (partir, sortir). $\mathrm{Du}$ fait que les constructions résultatives analytiques expriment le résultat sous la forme d'un constituant séparé du verbe, elles ont été considérées comme l'apanage des langues de type satellite-framed, telles que l'anglais ou le néerlandais. Toutefois, plusieurs auteurs ont cru reconnaître des constructions résultatives même dans les langues romanes (IT : Napoli 1992 ; FR : Legendre 1997 ; Muller 2001 ; Burnett/Troberg 2014 ; ESP : Martínez Vázquez 1998 ; Rodríguez Arrizabalaga 2016 ; ROUM : Farkas 2009, 2011 ; voir aussi l'aperçu dans Rodríguez Arrizabalaga 2016, 61), langues pourtant réputées verb-framed, ce qui met à mal les généralisations de Talmy dans leur application au domaine résultatif. En voici un exemple du roumain (Farkas 2011,69) :
(3) Fata
a frecat podeaua lună / oglindă.
fille-ART.DEF. a frotté sol-ART.DEF. lune/miroir
'La fille a frotté le sol pour qu'il devienne aussi propre / brillant que la lune / un miroir.'

Parmi ces constructions rétives à la généralisation de Talmy on trouve les verbes de changement chromatique, qui, dès lors, s'invitent toujours dans les études sur les

2 Elles sont analytiques dans la mesure où le résultat du procès n'est pas incorporé dans la forme verbale (p. ex. rougir 'devenir rouge').

3 Il n'est pas surprenant de trouver des contextes de transition (bridging contexts) dans lesquels le résultat est encore lié à un mouvement dans l'espace : néerl. Hij dronk zijn rivaal onder de tafel (lit. 'Il buvait son rival sous la table'). 
constructions résultatives. Comme on ne peut pas nier l'existence de verbes de changement chromatique dans les langues romanes, certains linguistes ont trouvé dans la nature morphosyntaxique du complément résultatif $S X$ un argument pour les qualifier de fausses résultatives. Seuls les SX adjectivaux entreraient en ligne de compte (cf. le constat de Farkas 2009, 59), disqualifiant d'entrée de jeu le français hexagonal, qui semble toujours avoir besoin d'une préposition $(e n)$ pour réaliser le changement d'état :

(4) a. *Il peint la porte verte.

b. Il peint la porte en vert.

Or, malgré l'importance de la nature du SX résultatif - puisque celui-ci fait office de ligne de partage typologique -, force est de constater que l'on n'a jamais exploré en détail les réalisations formelles des SX. ${ }^{4}$ Il suffit toutefois d'une recherche de corpus pour montrer que les verbes de changement chromatique en français puisent en réalité dans un inventaire de réalisations morphosyntaxiques beaucoup plus vaste. Même le contraste entre SAdj et SPrép s'en trouve relativisé.

Dans cette contribution, nous proposons dès lors de montrer que la construction résultative dans son application aux verbes de changement de couleur couvre une réalité plus diversifiée, à tel point qu'elle risque d'hypothéquer la recherche de 'la' construction résultative - en tant que schéma argumental plus abstrait (cf. aussi Boas 2003) -, ainsi que les tentatives de répartir les langues en fonction de l'opposition de Talmy (1991, 2000). Plus concrètement, nous entendons combattre trois idées (reçues) :

i. le français ne tolère jamais les SAdj résultatifs ; nous verrons qu'on en trouve, notamment si certaines conditions sont remplies (section 3 );

ii. les SPrép résultatifs se construisent toujours avec en en français; nous montrerons que la gamme des prépositions est beaucoup plus large, même si toutes les prépositions n'introduisent pas le même type de complément (section 4);

iii. en cas de concurrence entre les prépositions, la variation est libre ; nous verrons, notamment à propos du contraste $e n / d e$, que le choix est en grande partie motivé, même si les facteurs sous-tendant ce choix sont complexes (section 5).

Nous aborderons ces trois questions dans les sections 3 à 5 , après avoir explicité certains aspects méthodologiques de cette recherche (section 2). En section 6, nous ferons le point sur nos observations pour projeter toutes ces constructions sur une échelle qui les situe par rapport au prototype de la construction résultative.

4 Cela vaut même pour les langues germaniques. Celles-ci admettent souvent les deux types de SX, adjectival et prépositionnel, sans qu'on écarte pour autant les réalisations prépositionnelles du domaine résultatif. Si la différence est jugée pertinente pour les langues romanes, n’aurait-elle pas non plus une certaine pertinence pour les langues germaniques? 


\section{Corpus et méthode d'analyse}

Comme il s'agissait de mettre en carte l'éventail des constructions de changement chromatique (et non pas le paradigme des verbes ; voir Bernez 2014), nous avons limité notre recherche aux deux verbes peindre et colorer, qui feront l'objet d'un examen en profondeur, à partir d'une recherche lexicale dans le corpus Frantext (non catégorisé). Ces deux verbes ont été sélectionnés en raison de leur fréquence, ainsi que de la complémentarité de leurs schémas diathétiques (cf. l’opposition reconnue par Bernez 2014, 157). Le verbe peindre est emblématique de la classe éponyme des paint-verbs et entre prototypiquement dans la construction transitive à sujet agentif (humain). Parallèlement, colorer apparaît dans la construction transitive ('donner une/des couleur(s)') précédé d'un sujet humain ou inanimé, autorisant aussi des emplois pronominaux neutres dans lesquels il signifie 'prendre une couleur'.

Les exemples proviennent d'une recherche lexicale dans la version non catégorisée de Frantext (sans poésie), un corpus littéraire, moyennant la fonction de lemmatisation (expressions de recherche : \&'ccolorer ; \&cpeindre). Les attestations les plus récentes ont été parcourues jusqu'à ce qu'un échantillon provisoire de 200 exemples pertinents ait été obtenu pour chaque verbe. ${ }^{5}$ Notons que seuls les SX comportant un terme 'chromatique' - au sens large : des adjectifs et noms de couleur, des noms abstraits comme rougeur, ou encore, des colorants tels que bleu de méthylène - ont été retenus. En outre, nous avons finalement exclu les quelques exemples dans lesquels le COD de peindre n'est pas un objet affecté (du type peindre la porte en rouge) mais un objet effectué (peindre qqch en/de bleu sur le mur), réalisé sous forme de 'thème cumulatif' (incremental theme) (Dowty 1991), au cours de l'action :

(5) À son coin, un débit de boissons faisait aussi épicerie, à l'enseigne du Père Aimable, peinte de bleu au fronton [...]. (Garat A.-M., L'enfant des ténèbres, 2008, 170 sq.) ${ }^{6}$

En clair, ici il ne s'agit pas d'un mur ou d'une porte - objets préexistant à l'action - qui est couvert de peinture mais d'un objet, à savoir une enseigne, qui est créée par l'acte même de peindre. Cet objet est une représentation picturale qui naît à travers l'action. Il en est de même dans l'exemple suivant, relevant du domaine figuré. Dans peindre ('représenter') le monde en noir, la représentation (négative) du monde apparaît au cours de l'action :

(6) Et ne va pas écouter ta tante, qui te peindra le monde en noir. (Garat A.-M., Dans la main du diable, 2006, 62 sq.)

Ainsi, plusieurs exemples ont finalement été écartés (voir aussi 3.1), ramenant les totaux à respectivement 180 (peindre) et 189 (colorer).

5 Si pour peindre nous avons pu nous arrêter en 1984, pour colorer, moins fréquent, il a fallu remonter jusqu'à l'année 1899 .

6 Cet exemple et tous les exemples cités par la suite proviennent de Frantext. 


\section{Le biais résultatif du verbe et les SAdj résultatifs}

Il convient tout d'abord d'attirer l'attention sur le fait que cette recherche fournit quelques indices qui semblent nuancer la thèse selon laquelle les verbes de changement chromatique ne se construisent jamais avec un adjectif en français. Cette observation peut être mise en rapport avec un constat plus général et assez inattendu concernant l'usage de ces constructions : le verbe y présente un biais aspectuel résultatif.

3.1 Les SX adjectivaux résultatifs : débroussaillage du terrain

Les études consacrées aux verbes de changement chromatique en français ne reconnaissent pas l'existence de SX adjectivaux (Dagnac 2009 ; Vénérin-Guénez 2013). Seuls les Belges Grevisse/Goosse (2011, 438) ont remarqué leur existence, les reléguant toutefois au statut de régionalisme typique du français régional du Nord et de l'Est de la France et de la Belgique. On y retrouve, par exemple, la structure peindre vert, qui, selon Grevisse/Goosse, est souvent considérée comme un calque des langues germaniques. Ils ne se prononcent pas sur l'analyse du tour (ellipse ? épithète détachée exprimant le résultat ?).

Avant de regarder de plus près les exemples du corpus, il convient de signaler qu'à cause de la postposition (obligatoire) de l'adjectif de couleur en emploi adnominal (donc comme épithète), celui-ci se distingue parfois difficilement d'un adjectif en emploi attributif, le seul emploi qui nous intéresse ici, comme le montrent (7a et b), provenant du corpus Frantext :

(7) a. J'ai peint une première tête toute rouge avec ma nouvelle méthode que j'ai mise au point (Morgiève R., Ma vie folle, 2000, 26)

b. une nuit récente ensuite j'ai peint une tête bleue et j'ai retouché la tête rouge avec du bleu (Morgiève R., Ma vie folle, 2000, 26)

Seul le contexte, qui oppose ici une tête rouge et une tête bleue, permet de séparer les deux cas de figure. En outre, construit en épithète, le participe du verbe (colorés) peut être accompagné d’adjectifs apposés, qui en précisent le sens (gris, noirs, fleuris) :

(8) Suivent des rangs colorés, gris, noirs, fleuris, et encore des caddies avec bébés et verdure, puis la camionnette. (Thérame V., Bastienne, 1985, 109)

(9) Seules les oreilles restèrent roses ; par contraste, elles parurent extraordinairement colorées, rouge vif. (Vailland R., Drôle de jeu, 1945, 145)

L'exemple suivant est encore plus délicat. Le verbe peindre (au participe) prend pour COD un objet effectué (peindre la maison 'représenter la maison sous forme picturale'),

7 Les exemples (7)-(10) ne sont pas inclus dans notre corpus. 
c'est-à-dire un référent qui est réalisé au cours de l'action et qui ne préexiste donc pas à celle-ci :

(10) j'ai offert ce tableau à une amie. Aujourd'hui seulement [...] je me rappelle l'avoir peinte également, toute noire et labyrinthique, en train de flamber. (Bienne G., Le silence de la ferme, 1986, 62)

À l'instar de l'exemple (5), nous l'avions donc exclu. Il convient cependant de s'y attarder un peu dans la mesure où il illustre bien la nature dépictive (et donc non résultative ; Himmelmann/Schultze-Berndt 2005) de l'attribut adjectival. L'attribut du COD est composé de deux constituants juxtaposés, l'un de nature adjectivale (toute noire et labyrinthique), l'autre de nature infinitivale (et précédé de la locution aspectuelle en train de), qui décrivent l'état dans lequel se trouve l'objet. Le fait que l'attribut contienne un infinitif exclut la lecture résultative.

Après ce premier débroussaillage qui a permis d'écarter tous ces cas que l'on ne saurait considérer comme des exemples de constructions résultatives, il reste à peine un exemple de peindre + adjectif (ou nom) de couleur :

(11) Son Wrangler trop serré fait retomber sur la ceinture un bourrelet de graisse. Elle s'est peint les lèvres rose-bonbon. (Lasaygues F., Vache noire, hannetons et autres insectes, 1985, 177 sq.)

Pour colorer, on trouve cinq attestations résultatives, dont deux dans la même phrase : ${ }^{9}$

(12) Le malade est très amaigri, le visage coloré jaunâtre ; le ventre très volumineux, fait saillie. (sans auteur, Encyclopédie médicale Quillet : nouvelle encyclopédie pratique de médecine et d'hygiène, 1965, 153)

(13) Il y avait contre le mur toute une rangée de Spiridion. Le premier, tout en pastilles colorées vertes et rouges avec des yeux parme et des cheveux carotte. (Perry J., Vie d'un païen, 1965, 192 ; nous reprenons cet exemple ambigu ci-dessous)

(14) mais gris, tout pâlis, comme le reste de sa figure ... Il avait plus que les rides qu'étaient colorées foncé $[\mathrm{sic}],{ }^{10}$ par sillons du nez vers la bouche ... (Céline L. F., Mort à crédit, 1936, 774)

(15) considérons une sensation colorée rouge affectant un certain point de la rétine ; et d'autre part une sensation colorée bleue affectant le même point de la rétine (Poincaré H., La valeur de la science, 1905, 91)

8 L'interprétation selon laquelle elle a peint ses lèvres déjà rose-bonbon en une couleur non spécifiée semble peu plausible dans le contexte et est même exclue pour des raisons syntaxiques : l'emploi épithète aurait imposé le déterminant possessif en cas de modification par un adjectif (elle avait peint ses lèvres rose-bonbon).

9 L'exemple suivant n'a pas été retenu : qu'est-ce qu'un colorant ?[..] le trinitrobenzène est coloré / jaune /, mais il n'est pas colorant (Husson R. / Graf F., Manuel de biologie générale [...], 1965, 12). Les barres obliques correspondent en effet à des parenthèses, comme le montre la citation dans le TLFi (s. v. trinitrobenzène): coloré (jaune).

10 Notons l'orthographe foncées dans la version sur Google Books (datée de 2014), qui semble témoigner d'une hésitation entre une analyse adverbiale (une façon de colorer) et adjectivale (<https://books.google. be/books?isbn=2072447372>, dernier accès : 12/05/2018). Le site ne nous fournit pas d'informations plus précises concernant la date de l'édition numérisée. 
S'agirait-il d'un régionalisme lié à la proximité géographique des langues germaniques, comme le fait remarquer Le Bon Usage (Grevisse/Goosse 2011) ? Les informations sur l'appartenance géographique que nous avons réunies ne permettent pas de trancher : Frédéric Lasaygues était originaire de la banlieue sud de Paris (cf. Le Monde, 22/12/2010).." Toutefois, traducteur de l'anglais et fortement imbibé de la vie et de la culture américaines, on ne peut pas exclure une influence de la syntaxe anglaise sur son idiolecte. Poincaré, le célèbre mathématicien/physicien, après une jeunesse à Nancy (assez proche de la frontière franco-allemande, mais faisant partie du lorrain roman), a passé la plus grande partie de sa carrière à Paris. ${ }^{12}$ Céline quant à lui a vécu à Paris (et sa banlieue) et en Bretagne, mais a connu quelques séjours en Allemagne et en Angleterre pendant sa jeunesse (avant d'être exilé en Allemagne, mais cette période date d'après la parution de Mort à crédit). ${ }^{13}$ La vie de Jacques Perry s'est déroulée en grande partie dans la région de l'Île de France. ${ }^{14}$

L’origine géographique étant loin d'être concluante, il nous semble que la clé du mystère réside dans certains facteurs contextuels qui amenuisent le contraste entre résultatif et dépictif, comme il sera montré ci-dessous.

3.2 L'aspect grammatical du verbe et la syntaxe adnominale comme catalyseurs

Dans les 5 attestations de colorer + adj. (ex. (12)-(15)), le verbe apparaît sous sa forme participiale, construite en épithète d'un nom. Dans l'exemple (14), le participe se combine avec l'auxiliaire du passif, être. Dans les deux cas, on peut faire état d'un sens passif-résultatif, car seuls les verbes transitifs passivés (ainsi que les verbes inaccusatifs) peuvent construire leur participe en épithète (Legendre 1989). On peut donc conclure que c'est l'aspect passif-résultatif du verbe qui enlève la contrainte qui interdit aux verbes de changement chromatique de se combiner avec un adjectif pour marquer l'état résultant du procès. Le mécanisme pourrait être décrit comme suit. En utilisant le verbe à sa forme passive-résultative, on atténue l'impact de l'adjonction d'un état chromatique résultant en transformant celui-ci en état concomitant, ce qui aboutit à une configuration aspectuelle plus proche de la construction dépictive, qui, on le sait, est plus en phase avec le système français. L'aspect résultatif du verbe prépare donc mieux le verbe - on pourrait même parler de coercition aspectuelle (Lauwers/Willems 2011) - à accueillir un SX adjectival explicitant le résultat :

(16) a. X colore une sensation (changement d'état en cours) + bleue (résultat)

b. La sensation est colorée (changement d'état accompli) + bleue (description du résultat, selon le mode dépictif) 
Sans l'adjectif, dans (16a) le changement (perfectif) ${ }^{15}$ est encore en cours, alors qu'en (16b) il a déjà atteint son terme. Dans ce cas le verbe n'encode pas uniquement le changement d'état ( $X$ casse $Y$ en morceaux, $X$ peint $Y$ en rouge) dont l'état résultat est explicité par le SX résultatif, mais contribue aussi de façon non négligeable à l'expression du résultat. Il en résulte que l'aspect résultatif du verbe atténue l'impact de l'adjonction de l'adjectif. On pourrait même dire que le français n'accepte l'adjectif résultatif qu'à condition de le faire fonctionner dans une configuration aspectuelle se rapprochant de la configuration dépictive.

Toujours dans le cas de colorer, la forme participiale ne réduit pas seulement l'écart par rapport à la configuration dépictive par sa valeur aspectuelle, mais aussi par sa syntaxe adnominale, qui fonctionne comme un deuxième co-facteur légitimant le SX adjectival. En effet, l'emploi épithète entraîne la juxtaposition du participe avec d'autres adjectifs de couleur, qui pourraient être interprétés comme des adjectifs apposés au participe coloré $:^{16}$

(17) Le premier, tout en pastilles colorées vertes et rouges avec des yeux parme et des cheveux carotte. (Perry J., Vie d'un païen, 1965, 192)

Dans cette lecture, ces adjectifs décriraient de manière plus précise la couleur de pastilles colorées, étant donné que le participe coloré exprime un état chromatique vague ou générique qui demande à être précisé par des appositions (dépictives donc). On peut supposer que l'ambiguité de ce 'bridging context' facilite la construction $\mathrm{V}+$ adjectif.

Reste le seul exemple comportant le verbe peindre. Là encore, le verbe se retrouve à sa forme participiale, certes dans une configuration pronominale réfléchie. Comme la construction pronominale réfléchie peut être considérée comme une reformulation d'une construction transitive ( $X$ peint les lèvres [de X]) - dont le sujet est désormais co-référentiel avec le COI datif $(s e)$ - on ne saurait ranger ces constructions parmi les passives. Toutefois, sur le plan aspectuel, le participe passé de cette forme active peut prendre une valeur accomplie (cf. on a rempli le verre $\rightarrow$ le verre est plein maintenant), même si sa valeur antérieure est, certes, plus fréquente :

(18) Elle s'est peint les lèvres rose-bonbon. $\rightarrow$ Voilà que ses lèvres sont rose-bonbon maintenant. (Lasaygues F., Vache noire, hannetons et autres insectes, 1985, 177 sq.)

Ici encore, c'est l'aspect résultatif du verbe qui ouvre la voie à l'adjectif.

En définitive, il nous semble donc qu' il faudrait relativiser la contrainte sur les constituants résultatifs de nature adjectivale. Certes, l'adjectif attribut résultatif reste un phénomène très rare, voire 'contre nature' en français hexagonal, ce qui fait que seulement 2 des 4 locuteurs natifs que nous avons consultés l'acceptent (en donnant 4 sur 5 ) dans les conditions indiquées. Il n'empêche qu'il est attesté si le contexte atténue 'le choc' causé par l'adjonction d'un adjectif, en se présentant davantage sous l'aspect d'une configu-

$15 X$ colore cette substance \{pendant des heures ??/en 2 heures\}.

16 Configuration que nous avons exclue de l'analyse à chaque fois que l'apposition était marquée par une virgule (cf. supra section 3.1). 
ration dépictive. L'importance de ces facteurs se reflète aussi dans les jugements des locuteurs natifs qui donnent systématiquement un score moins élevé aux emplois actifs.

Avant de clore ce chapitre, il importe de signaler que, de manière plus générale, peindre et colorer, même avec des SPrép, font preuve d'une remarquable préférence pour l'emploi passif périphrastique, qui, comme on le sait, est lié à une visée résultative (voir infra section 5.2) :

Tableau 1 : Préférence globale pour la visée passive résultative

\begin{tabular}{|l|c|c|c|c|c|c|c|c|}
\hline \multirow{2}{*}{} & \multicolumn{2}{|c|}{ actif } & \multicolumn{2}{c|}{ passif } & \multicolumn{2}{c|}{ autre $^{17}$} & \multicolumn{2}{c|}{ total } \\
\cline { 2 - 9 } & $\#^{18}$ & $\%$ & $\#$ & $\%$ & $\#$ & $\%$ & $\#$ & $\%$ \\
\hline colorer & 64 & 33,86 & 86 & 45,50 & 39 & 20,63 & 189 & 100 \\
\hline peindre & 38 & 21,11 & 139 & 77,22 & 3 & 1,67 & 180 & 100 \\
\hline
\end{tabular}

Ces chiffres dépassent de loin la fréquence 'normale' du passif périphrastique en français, comme ceux fournis par McLaughlin (2010), par exemple, qui varient de 1,6\% à $22 \%$ selon le registre.

Ces profils distributionnels pour le moins remarquables appellent une explication. On pourrait y voir le reflet d'une pression typologique dans la mesure où le français peine à exprimer des changements d'état à travers une construction résultative analytique. C'est pourquoi les locuteurs sont amenés à utiliser des formes aspectuelles accomplies pour atténuer le conflit typologique entraîné par l'adjonction d'un SX résultatif, fût-il un SPrép. C'est comme si le français appelait le verbe à la rescousse pour prêter main forte à la préposition dans son rôle de marque facilitant la réalisation du SX résultatif. ${ }^{19}$

\section{Les compléments (prépositionnels) de nature instrumentale}

La recherche lexicale (et lemmatisée) que nous avons menée ( peindre $_{\text {lemme }}$ colorer ${ }_{\text {lemme }}$ ) a permis de repérer la liste suivante de prépositions qui accompagnent les verbes de changement chromatique :

17 Pronominal emploi neutre, pronominal passif, pronominal réfléchi.

18 Le symbole \# renvoie au nombre absolu de cas attestés dans le corpus.

19 Il en découle une hypothèse facile à tester : les langues germaniques devraient afficher un biais résultatif moins fort. 
Tableau 2 : Prépositions

\begin{tabular}{|c|c|c|c|c|c|c|c|c|c|c|c|c|c|c|c|c|}
\hline & \multicolumn{2}{|c|}{$\grave{a}$} & \multicolumn{2}{|c|}{ avec } & \multicolumn{2}{|c|}{ dans } & \multicolumn{2}{|c|}{ de } & \multicolumn{2}{|c|}{ en } & \multicolumn{2}{|c|}{ par } & \multicolumn{2}{|c|}{ selon $^{20}$} & \multicolumn{2}{|c|}{ total } \\
\hline & $\#$ & $\%$ & \# & $\%$ & \# & $\%$ & \# & $\%$ & \# & $\%$ & \# & $\%$ & \# & $\%$ & \# & $\%$ \\
\hline colorer & 9 & 4,89 & 1 & 0,54 & - & - & 55 & 29,89 & 113 & 61,41 & 5 & 2,71 & 1 & 0,54 & 184 & 100 \\
\hline peindre & 2 & 1,12 & 6 & 3,35 & 1 & 0,56 & 28 & 15,64 & 142 & 79,33 & - & - & - & - & 179 & 100 \\
\hline total & 11 & 3,03 & 7 & 1,93 & $\mathbf{1}$ & 0,28 & 83 & 22,87 & 255 & 70,25 & 5 & 1,38 & $\mathbf{1}$ & 0,28 & 363 & 100 \\
\hline
\end{tabular}

Dans ce qui suit, nous relèverons d'abord des constructions prépositionnelles (avec, à, par) dans lesquelles le changement de couleur n'est pas exprimé directement (il peut tout simplement être inféré), avant d'aborder en section 5 le contraste entre en et de. Notons que ces prépositions introduisent des régimes nominaux, mais ceux-ci diffèrent d'une préposition à l'autre : alors que à, avec, dans, par et selon se combinent uniquement avec des SN, en et de accompagnent le plus souvent des noms nus (i.e. sans déterminant).

\subsection{Avec}

En étant accompagnés d'un complément prépositionnel introduit par avec et suivi d'un SN complet, peindre (6 ex.) et colorer (1 ex.) se construisent avec un complément adverbial qui conçoit la couleur sous l'angle de son rôle instrumental. La couleur, en tant que matériel et non pas nuance de couleur, toujours au pluriel dans notre corpus y fonctionne en quelque sorte comme un moyen ou un instrument chromatique :

(19) entre des maisons longues, étirées, médiévales et baroques mêlées, peinte avec des couleurs tendres, italiennes, aux fenêtres chargées de fleurs bleues, de cages à oiseaux (Rambaud P., La Bataille, 1997, 27 sq.)

L'application de cet instrument provoque un changement chromatique. À cet effet, la construction en avec exploite la sous-détermination sémantique qui sous-tend le nom couleur et les adjectifs convertis en noms de couleurs. En effet, la couleur y exprime à la fois la substance colorante et la couleur (résultante) :

(20) ces tas sont colorés avec des ocres ou des oxydes (Collectif, Arts et littérature dans la société contemporaine, dir. Pierre Abraham, t. 1, 1935, 2011)

(21) On peint avec du blanc. (Queneau R., Journaux 1914-1965, 1996, 729 sq.)

20 Dans un seul exemple on trouve un complément introduit par selon qui renvoie à une espèce de modèle qui est suivi de la manière dont le coloriage s'effectue (ici au figuré) : Mais dès que je me mets au travail, tout se colore selon mes couleurs éternelles; mes personnages les plus beaux entrent dans une certaine lumière. (Mauriac F., Journal 2, 1937, 155) 
Curieusement, 3 des 7 exemples comportent des modificateurs qui pointent vers un emploi métaphorique du nom couleurs :

(22) entendre le chef de notre provisoire gouvernement peindre notre dénuement avec d'aussi sombres couleurs (Pouquet J., Journal sous l'Occupation en Périgord : 1942-1945, 2006, 167 sq.)

Notons que le complément en avec est indépendant d'un objet qui change de couleur, comme le montre l'emploi absolu du verbe en (21) et dans l'exemple (23), qui est antérieur à l'échantillon que nous avons examiné exhaustivement :

(23) Aujourd'hui les artistes peignent avec des couleurs plus fluides. (Collectif, Arts et littérature dans la société contemporaine, dir. Pierre Abraham, t. 1, 1935, 3004)

En outre, cette construction n'exprime pas nécessairement un changement d'état chromatique. Ainsi, la couleur des tas en (20) n'est pas oxyde après un traitement avec des oxydes. On peut en déduire que la construction en avec n'est pas une construction résultative qui attribue un résultat résultatif à un support réalisé sous la forme d'un COD. Il s'agit plutôt d'un complément adverbial de moyen ou d'instrument.

Le constat selon lequel avec est presque toujours suivi du nom couleurs au pluriel ( 5 occurrences sur 7 ) est sans doute lié au fait que cette construction ne marque pas vraiment un état chromatique résultant. En effet, nous allons voir que l'état chromatique est le plus souvent caractérisé par son unicité (cf. section 6). En revanche, le procès encodé par $\mathrm{V}+$ avec ne connaît pas cette contrainte d'unicité : ce qui compte, c'est qu'un agent (le sujet) ait appliqué des substances colorantes sur un support. Certes, la construction en avec se rapproche du changement chromatique par le biais de la sous-détermination sémantique que caractérise les adjectifs/noms de couleurs, qui dénotent à la fois la substance chromatique et l'état chromatique résultant. De plus, là où cette sous-détermination n'existe pas (p. ex. oxyde), l'énoncé se voit enrichi d'implicatures conversationnelles selon lesquelles l'application d'une substance chromatique provoque aussi un changement de couleur.

\section{$4.2 \grave{A}$}

La deuxième construction incluant un complément prépositionnel avec à est une variante de la première :

(24) un transatlantique en génoise, avec le pont tout en nougatine, et une mer en crème chantilly colorée au curaçao bleu (Vergne A., L'Innocence du boucher, 1984, 61)

(25) Les chondriosomes seront colorés au vert janus $B$ et le 'noyau' vitellin au rouge neutre ou au bleu de crésyl brillant. (Husson R. / Graf F., Manuel de biologie générale à l'usage des travaux pratiques. 1. La Cellule, 1965, 60)

Le complément exprime le moyen-instrument, le plus souvent un colorant classique (coloré avec du vert janus, du rouge neutre ou du bleu de crésyl), ou encore, une substance 
colorante 'ad hoc', telle que le curaçao bleu. Tout comme la construction en avec, cette construction s'applique aussi à d'autres types de noms que les noms chromatiques, qui n’ont pas été relevés dans l'échantillon :

(26) On se demande de quoi est fait ce pâté de son, sans gras ni viande, coloré au jus de betterave. (Garat A. M., Les mal famées, 2000, 132)

Toujours à l'instar de avec, à se construit avec un SN comportant le mot couleurs au pluriel (3 ex. sur 11) :

(27) le VAB peint aux couleurs d'oie blanche de l'ONU (Rolin O., Tigre en papier, 2002, 129 sq.)

Le SN qui suit la préposition est introduit par un article défini en emploi non spécifique $(\grave{a}+l e=a u):$ au carmin acétique, au henné (3 ex.), au curaçao bleu, aux couleurs X (3 ex.), au rouge neutre ou au bleu brillant de crésyl, au vert janus, au bleu de Prusse. Cette contrainte de non-spécificité n'est pas surprenante dans la mesure où c'est la marque de fabrique de la préposition à :

(28) la fille au chapeau /vs/ la fille avec le chapeau

(29) des moteurs à combustion interne alimentée au propane /vs/ avec du propane

(30) une sauce au curry /vs/ avec du curry

Notons toutefois quelques différences par rapport à avec. Tout d'abord, le traitement 'colorant' exprimé par à est moins conceptualisé comme le résultat 'occasionnel' de l'application d'une substance à travers l'intervention d'un agent externe que comme une caractérisation 'interne', plus intrinsèque du procès verbal. Le complément introduit par à est donc plus proche d'un complément de manière, d'une façon de colorer. C'est ce qui explique aussi des exemples tels que peint aux couleurs d'oie blanche de l'ONU (27) dans lesquels la dimension instrumentale est plus ou moins absente et cède le pas devant la caractérisation (cf. Cadiot 1991). On retrouve d'ailleurs le même effet de caractérisation intrinsèque dans alimentée au propane (29) et une sauce au curry (30). De même, dans la fille au chapeau (28), il se pourrait même que la fille porte toujours un chapeau (caractérisation intrinsèque). On peut y voir le résultat de l'emploi non spécifique de l'article défini qui n'individualise pas le nom qui suit. Si on applique ce raisonnement aux verbes de changement chromatique, on voit que le moyen (la substance active) est désindividualisé au profit d'une lecture caractérisante du procès, c'est-à-dire d'une façon de colorer.

Une deuxième différence entre les SPrép introduits par avec et par à tient à la diathèse du verbe. Si dans 5 cas sur 9, avec se construit avec un verbe ayant une forme active (21)(23), il en est tout autrement de $\grave{a}$, qui ne se trouve à l'actif que dans un exemple sur 11 (31):

(31) les cornemuses de Macbeth colorent la nuit aux couleurs de l'Espagne ou de l'Ecosse des guerres royales (Serrière M. T., Le T.N.P. et nous, 1959, 125)

Cet exemple confirme cependant le biais décausatif (ou déagentif) de la construction en à dans la mesure où l'agent est inanimé, ce qui correspond au sens figuré du verbe. 
D'autre part, la construction en à présente une certaine analogie avec la construction en par, comme le montre l'exemple (32) trouvé dans le même texte :

(32) Ces enclaves cellulaires se colorent vitalement par le rouge neutre ou le bleu de crésyl brillant. (Husson R. / Graf F., Manuel de biologie générale à l'usage des travaux pratiques. 1. La Cellule, 1965, 49)

Cet exemple suggère que l'action de la substance colorante peut également être conceptualisée au moyen d'un complément d'agent (ou d'instrument, cf. infra section 4.3).

$\mathrm{Si}$ on combine maintenant toutes les pièces du puzzle, on aboutit à un contraste entre deux configurations diathétiques (l'exemple (31) étant un cas intermédiaire avec un sujet inanimé) :

[AGENT] [PROCÈS] [PATIENT] [avec MOYEN individualisé]

[PATIENT] [PROCÈS] [à AGENT non individualisé, tendant vers la caractérisation du procès]

En d'autres mots, la construction en à tend à s'aligner sur un schéma diathétique passif, caractérisé par la récession actancielle du sujet agentif. On serait tenté d'établir ici une analogie avec la construction en par, analogie qui n'est pas sans rappeler qu'autrefois la préposition à pouvait introduire des compléments d'agent. Le français moderne en a gardé d'ailleurs quelques traces figées : mangé aux mites/aux vers (Grevisse/Goosse 2011, 420 sq. ; Gaatone 1998, 198).

4.3 Par

On retrouve également 5 attestations de colorer suivies de par $+\mathrm{SN}$ plein :

(33) Coupe voisine de la précédente traitée par la réaction de substitution au calcium puis colorée par la safranine et le vert lumière. (Husson R. / Graf F., Manuel de biologie générale à l'usage des travaux pratiques. 1. La Cellule, 1965, 87)

Dans tous les cas, par introduit une substance colorante active qui provoque un virage chromatique. Dans un exemple, le changement chromatique est dû à une autre cause, à savoir une rougeur qui apparait sur le visage :

(34) avec son visage fermé, absent, coloré par une rougeur que le froid ne suffit pas à expliquer complètement (Simon C., Les Géorgiques, 1981, 95)

Tout comme à, par présente un biais clairement décausatif/déagentif : trois passifs, un emploi pronominal passif et un emploi pronominal neutre (cf. section 5.2.2) :

(35) Leur protoplasma renferme en abondance des grains qui se colorent avec intensité par les couleurs acides, l'éosine ou l'orange. (Calmette A., L'Infection bacillaire et la tuberculose chez l'homme et chez les animaux, 1920, 115)

La cooccurrence avec le passif pronominal suggère que ce complément en par n'est peutêtre pas encore un véritable complément d'agent mais un complément d'instrument 
(p. ex. un plan se juge par ses résultats, exemple fourni par Togeby 1982, t. 1, 422, cité dans Melis 1990, 93). En effet, tous les observateurs semblent d'accord pour affirmer que le tour médio-passif ne prend pas de complément d'agent (Melis 1990, 93 et les références citées par celui-ci), sauf en cas de focalisation restrictive (ne ... que par $X$ ), par exemple, qui établit une opposition paradigmatique (Melis 1990, 94). Notons toutefois qu'ici le complément d'instrument peut devenir le sujet du verbe dans la construction active (à l'instar du complément d'agent), mais cela est une propriété spécifique du verbe colorer qui accepte ce genre d'alternance (la safranine colore X), cf. Bernez (2014, 180).

\section{Les compléments résultatifs en vs $d e$ : facteurs déterminant le choix}

Après avoir passé en revue les constructions instrumentales (avec, par, à) qui ne marquent qu'indirectement le changement de couleur, il importe de regarder de plus près les deux prépositions les plus fréquentes (cf. tableau 2), en (et sa variante combinatoire dans) et de, qui, elles, marquent bel et bien un véritable changement chromatique. Que de figure ici aux côtés de en - la seule préposition reconnue dans la littérature sur les constructions résultatives - a de quoi surprendre, sauf si on regarde la chose d'un point de vue typologique : en espagnol l'emploi de de est même plus fréquent que celui de en (Rodríguez Arrizabalaga 2016). Signalons toutefois que le fait que de, construction sans doute plus littéraire, soit si bien représenté peut avoir été influencé par le caractère littéraire du corpus. N'empêche que, de manière globale, en reste trois fois plus fréquent que de : 255 occurrences vs 83 (cf. tableau 2).

Avant d'aborder en détail la distribution de en et de, il importe de rappeler que la construction avec en alterne avec dans + SN, d'après une alternance qui est encore attestée ailleurs dans le système de la langue (un seul exemple dans tout le corpus) :

(36) plinthes et tête de lit, peintes, elles, dans un jaune plus soutenu (Carrère E., D'autres vies que la mienne, 2009, 93 sq.)

Dans ce qui suit, nous allons examiner de plus près les propriétés des deux constructions, celles introduites par en et par de, en identifiant les facteurs sous-tendant leur distribution, et donc, leur complémentarité. En gros, trois types de facteurs seront identifiés : la structure du SN régi par la préposition (section 5.1), la visée diathétique du prédicat (section 5.2) et l'affectation globale et exclusive du référent par le changement chromatique (section 5.3).

\subsection{La structure du $\mathrm{SN}$ régime de la préposition}

Un premier facteur qui détermine le choix entre de ou en est lié à la structure interne des $\mathrm{SN}$ dépendant de la préposition. Cette différence de structure n'est pas spécifique pour les verbes de changement chromatique, mais influence bel et bien les latitudes expressives du locuteur lorsque celui-ci décrit un changement de couleur. En effet, contraire- 
ment à de, la préposition en n'accepte pas facilement les SN pleins, c'est-à-dire les syntagmes comportant au moins un déterminant et un nom, accompagné le plus souvent d'un modificateur. Voir (37a) vs (37b) :

(37) a. de + SN plein : (peint/coloré) d'un beau jaune

b. ??en + SN plein : ??(peint/coloré) en un beau jaune

Les chiffres sont éloquents : de + SN plein ( 28 exemples, dont 27 au singulier) vs en + SN plein (2 exemples, au pluriel) :

(38) a. Peints en des ${ }^{21}$ couleurs malsaines [...]. (Romilly J. de, Les E Eufs de Pâques, 1993, 36 sq.)

b. se colorent à cette heure en des rouges de corail tout à fait étrangers aux paysages de nos climats (Loti P., Vers Ispahan, 1904, 908)

Cette contrainte structurelle imposée par en constitue un sérieux handicap quand le locuteur veut faire une description précise et subjective du résultat chromatique, dépassant la palette chromatique de base offerte par les noms de couleurs simples (vert) ou composés (rouge foncé, rouge brique). En effet, dans tous ces cas, l'insertion d'un déterminant s'impose ( ${ }^{*}$ en beau jaune), ce qui entre en conflit avec la contrainte syntaxique imposée par en.

Ce handicap de en par rapport à de joue dans différents cas de figure. Ainsi, nous avons relevé trois configurations dans les exemples de de + SN plein.

\section{(i) Les transferts taxonomiques ('une sorte de rouge')}

Tant en que de peuvent être suivis de noms nus (sans déterminant), accompagnés le cas échéant de modifications référant à des nuances chromatiques stéréotypées : peint en/de rouge vif, en couleur bronze, en couleur bleu-ciel. Or, dès que le locuteur veut indiquer des nuances plus 'ad hoc', il faut réaliser un transfert du massif au comptable par l'insertion obligatoire d'un déterminant, ce qui donne une lecture taxonomique ('sorte de, nuance de') :

(39) a. Elle avait les pommettes rouge vif, les yeux bordés de noir, et les lèvres peintes d'un bleu pâle violacé. (Aventin C., Le Cour en poche, 1988, 195)

Si on compare cette syntaxe avec la syntaxe autorisée par en, le contraste est net :

（39） b. ??en un bleu pâle violacé

Cela ne veut pas dire que en bloque tout transfert, notamment lorsqu' il se combine avec un pluriel, qui provoque également une lecture comptable (p. ex. peints en bleus de diverses tonalités tirant vers le violet).

21 L'on peut se demander, comme le remarque un lecteur, si l'apparition exceptionnelle de en + des (au pluriel donc) n'est pas légitimée ici par le fait que $d e+d e s \mathrm{~N}_{\text {pluriel }}$ est exclu pour des raisons d'euphonie. En effet, ce cas de figure déclencherait automatiquement une haplologie $\left(d e+\mathrm{N}_{\text {pluriel }}\right)$. 
(ii) Des SN pleins dénotant une quantité indéfinie de substance colorante

La deuxième configuration concerne un SN comportant un quantifieur (indéfini) :

(40) le rossolio de Turin à base de pétales de roses rouges additionnées de cannelle et de girofle, le tout coloré d'un peu de carmin (sans auteur, Les Grandes heures de la cuisine française par Cécile Éluard-Valette, 1964, 234)

(iii) Des SN pleins transformant un nom de propriété (donc abstrait) en une occurrence concrète

Pour le troisième ensemble d'occurrences de $d e+\mathrm{SN}$ plein nous nous référons à Kleiber (2011). C'est le cas du nom de propriété rougeur (plusieurs exemples) qui peut prendre une valeur concrète telle que 'tache, plaque rouge' :

(41) ses joues tannées [...] se crevassent de rides et se colorent d'une rougeur inattendue (Déon M., Le Balcon de Spetsai, 1961, 87)

5.2 L'impact de la diathèse décausative (déagentive) du prédicat

La concurrence entre de et en est également déterminée par la diathèse du verbe.

\subsubsection{L'impact de l'orientation [+/- agentive] des verbes}

Examinons d'abord si l'alternance de/en est déterminée lexicalement, par le choix du verbe peindre ou colorer. Rappelons d'abord que en est la préposition la plus fréquente pour les deux verbes. Toutefois, la proportion des exemples avec de semble varier d'un verbe à l'autre. Ainsi, la préposition de représente 32,74\% du total des occurrences de en et de pour colorer $(55 / 168)$ et seulement $16,47 \%$ pour peindre $(28 / 170)\left(\mathrm{X}^{2}=11.208, \mathrm{df}=\right.$ $1, \mathrm{p}<0.001)$. Si on écarte les emplois dans lesquels le verbe de changement chromatique fonctionne comme épithète ou apposition (une porte [] peinte en vert), pour ne retenir que les emplois comme verbe fini ou à l'infinitif - dans lequel le support (la porte) de la prédication chromatique apparaît comme un constituant autonome régi par le verbe chromatique -, les tendances se renforcent encore légèrement, comme le montre la comparaison des tailles d'effet (Cramer's V : 0.189 vs 0.202$): 35,35 \%$ pour colorer (35/99) et $14,29 \%$ pour peindre $(5 / 35)\left(\mathrm{X}^{2}=4.5211, \mathrm{df}=1, \mathrm{p}<0.05\right)$. Or, si on ne retient que les emplois à la voix active (verbe fini, infinitif et emploi pronominal réfléchi) pour éviter tout biais dû à la décausativisation provoquée par la diathèse verbale, on constate que le lexique en soi n'est pas un facteur prépondérant. En effet, le contraste entre ces deux types de verbes - l'un (peindre) étant agentif, l'autre pas ${ }^{22}$ - par rapport au choix de la

22 Rappelons à ce propos que peindre est un verbe agentif qui accepte le contrôle par un sujet agent, comme le montrent les tests classiques, par exemple les adverbes orientés sur l'agent: Il peint la porte en bleu avec une 
préposition $(e n / d e)$ n'est pas significatif $\left(\mathrm{X}^{2}=0.039994, \mathrm{df}=1, \mathrm{p}>0.05\right): 18,75 \%$ pour colorer $(12 / 64)$ et $14,29 \%$ pour peindre $(5 / 35)$. Bref, si à première vue on aurait donc pu croire à un biais lié au verbe, l'alternance entre de et en s'avère surtout régie par la diathèse verbale.

\subsubsection{L'impact de l'orientation [+/-agentive] du prédicat : le rôle de la diathèse}

Plus que les préférences lexicales (déterminées par l'orientation agentive ou non des verbes), c'est donc la diathèse décausative (ou désagentive) qui régit la distribution de la préposition de. En, quant à lui, est moins sélectif à ce propos. Par décausatif (ou déagentif) nous entendons les emplois passifs périphrastiques ( $X[e s t]$ coloré) et les formes pronominales ( $X$ se colore), à la fois en emploi passif et en emploi neutre. Dans tous ces cas, le sujet ne contrôle pas le procès. Voici les résultats chiffrés :

Tableau 3 : Choix de la préposition $(e n / d e)$ face à la diathèse et au verbe

\begin{tabular}{|l|c|c|c|c|c|c|}
\hline \multirow{2}{*}{} & \multicolumn{2}{|c|}{ actif } & \multicolumn{2}{c|}{ décausatif } & \multicolumn{2}{c|}{ total } \\
\cline { 2 - 7 } & $\#$ & $\mathbf{\%}$ & $\#$ & $\mathbf{\%}$ & $\#$ & $\mathbf{9}$ \\
\hline $\boldsymbol{d} \boldsymbol{e}$ & $\mathbf{1 7}$ & $\mathbf{2 0 , 4 8}$ & $\mathbf{6 6}$ & $\mathbf{7 9 , 5 2}$ & $\mathbf{8 3}$ & $\mathbf{1 0 0}$ \\
\hline colorer & 12 & 21,82 & 43 & 78,18 & 55 & 100 \\
\hline peindre & 5 & 17,86 & 23 & 82,14 & 28 & 100 \\
\hline en & $\mathbf{8 2}$ & $\mathbf{3 2 , 1 6}$ & $\mathbf{1 7 3}$ & $\mathbf{6 7 , 8 4}$ & $\mathbf{2 5 5}$ & $\mathbf{1 0 0}$ \\
\hline colorer & 52 & 46,02 & 61 & 53,98 & 113 & 100 \\
\hline peindre & 30 & 21,13 & 112 & 78,87 & 142 & 100 \\
\hline
\end{tabular}

Regardons d'abord la préposition de. Ainsi, dans 23 des 28 exemples $(82,14 \%)$ le verbe peindre figure à la voix passive. On pourrait donc dire que pour utiliser le verbe peindre avec un complément en $d e$, il faut d'abord le 'forcer' dans une construction décausative qui le prépare à ce type de fonctionnement. Les très rares emplois actifs de peindre de sont assez particuliers dans la mesure où le verbe y perd son sens 'pictural' fort, à savoir 'faire changer d'état chromatique en appliquant de la peinture'. En effet, dans trois exemples peindre signifie 'maquiller' :

(42) Sa belle bouche bien dessinée, aux lèvres pleines, elle la peignait de rouge. (Jenni A., L’Art français de la guerre, 2011, $491 \mathrm{sq.)}$

Dans un exemple, peindre se combine avec un sujet inanimé (donc sans contrôle) en emploi métaphorique (personnifié) : 
(43) La veilleuse peignait de bleu fade le lit et les murs, stylisant l'austérité de cette chambre. (Garat A.-M., Dans la main du diable, 2006, 723 sq.)

Enfin, un exemple est suivi du nom couleurs au pluriel, qui est un autre facteur légitimant l'emploi de de, au détriment de en (cf. section 5.3.1) :

(44) Il fit pour elle des jouets de bois qu' il peignit de couleurs vives. (Flem L., Lettres d'amour en héritage, 2006, 65)

Tout comme pour peindre de, 78,2\% des occurrences de colorer de sont liées à la visée décausative. La fréquence légèrement plus élevée - qui n’est pas du tout significative, du reste $\left(\mathrm{X}^{2}=0.018266, \mathrm{df}=1, \mathrm{p}>0.05\right)$ - de la voix active par rapport à peindre de ne contredit pas nécessairement l'hypothèse que nous sommes en train de défendre ici, car dans la plupart des occurrences de colorer à la voix active, la visée décausative est réalisée lexicalement par le verbe et son sujet. En effet, dans 10 des 12 exemples du verbe à la voix active, les sujets s'avèrent inanimés : p. ex. le sang afflua à sa figure [...] et lui colora d'écarlate jusqu'à la peau du crâne. Le seul contre-exemple à sujet humain s'avère assez particulier dans la mesure où la cause (par des injections) est exprimée par un nom inanimé remplissant la fonction de complément circonstanciel, qui relègue l'agent humain ( $j e)$ à l'arrière-plan, ce qui, indirectement, confirmerait l'hypothèse décausative $:^{23}$

(45) j'avais fait de l'île un parc, un salon, astiquant les grèves de nacres, polissant les récifs, colorant de rouge vif par des injections dans les racines des bosquets entiers (Giraudoux J., Suzanne et le Pacifique, 1921, 201)

En somme, l'examen de peindre de et de colorer de nous amène à la conclusion suivante : de ne semble pas approprié à l'expression du changement chromatique fort dans le cadre d'un procès agentif dans lequel un agent humain externe, réalisé dans la position sujet, intervient intentionnellement pour changer l'état chromatique d'un support.

Le profil de en, en revanche, est moins net. Pour peindre en, la visée décausative (emploi passif) domine également, tout comme nous l'avons vu pour peindre de : 112 occurrences sur 142 (78,87\%). Or, à la différence de peindre de, les 30 occurrences 'actives' de peindre en évoquent toutes un procès dans lequel un agent humain externe (29 sujets humains + un inanimé personnifié) provoque un changement de couleur (qui ne soit pas un simple maquillage). Colorer en, de son côté, se combine aisément avec les deux voix (53,98\% décausatif ; 46,02\% actif). Toutefois, $11,54 \%$ (6/52) seulement des occurrences 'actives' concernent un sujet humain, qui contrôle le changement chromatique (j'ai coloré une de ces ombres en jaune). Sur ce point, colorer en se distingue clairement de peindre en en contexte actif (non décausatif). En somme, en s'avère neutre par rapport à la problématique diathétique. À travers sa distribution transparait tout simplement le contraste lexical agentif (peindre) / non agentif (colorer). De même, le fait que la lecture décausative domine avec peindre en n'est que le reflet de la préférence globale du

23 L'autre exemple de colorer de à sujet humain comporte un SN modifié qui s'explique à la lumière du principe présenté sous 5.1. 
verbe peindre (en construction résultative) pour les constructions décausatives $\left(\mathrm{X}^{2}=\right.$ 5.51963.5766, $\mathrm{df}=1, \mathrm{p}<0.05)$; en n'y change rien :

Tableau 4 : Biais décausatif global de peindre

\begin{tabular}{|l|c|c|c|c|c|c|}
\hline \multirow{2}{*}{} & \multicolumn{2}{|c|}{ agentif } & \multicolumn{2}{c|}{ décausatif } & \multicolumn{2}{c|}{ total } \\
\cline { 2 - 7 } & $\#$ & $\%$ & $\#$ & $\%$ & $\#$ & $\%$ \\
\hline colorer & 65 & 34,39 & 124 & 65,61 & 189 & 100 \\
\hline peindre & 41 & 22,78 & 139 & 77,22 & 180 & 100 \\
\hline
\end{tabular}

Faisant la synthèse des sections 5.2.1 et 5.2.2, on peut conclure que l'emploi de la préposition de se marie parfaitement bien avec une visée décausative (déagentive), que celle-ci soit due à la diathèse du verbe ou à la nature inanimée des sujets (colorer). En, en revanche, n'est pas sélectif. D'une part, il est capable d'évoquer des événements agentifs, notamment avec peindre (3o ex.) et même avec colorer (6 ex.), mais ne s'oppose nullement à des configurations décausatives. En est donc une préposition neutre par rapport à la diathèse ; elle ne fait qu'écho à la distribution générale des deux verbes à ce propos. Voici, pour rappel, les chiffres globaux; le rapport entre le choix de la préposition et l'opposition diathétique est significatif (sans correction Yates), même sans que la variable sujet ne soit prise en considération $\left(\mathrm{X}^{2}=4.121078, \mathrm{df}=1, \mathrm{p}<0.05\right)$.

Tableau 5 : Biais décausatif global de la construction en de

\begin{tabular}{|l|c|c|c|c|c|c|}
\hline \multirow{2}{*}{} & \multicolumn{2}{|c|}{ agentif } & \multicolumn{2}{c|}{ décausatif } & \multicolumn{2}{c|}{ total } \\
\cline { 2 - 7 } & $\#$ & $\%$ & $\#$ & $\%$ & $\#$ & $\%$ \\
\hline $\boldsymbol{d e}$ & 17 & 20,48 & 66 & 79,52 & 83 & 100 \\
\hline en & 82 & 32,16 & 173 & 67,84 & 255 & 100 \\
\hline
\end{tabular}

Face à l'orientation décausative (et notamment passive) de la construction en de, on serait tenté de conclure que la préposition de introduit une sorte de complément d'agent, comme dans les constructions passives périphrastiques. L'analogie est d'autant plus pertinente que le complément d'agent en de passe pour une variante moins dynamique du complément en par: une soutenance suivie d'une réception vs un espion suivi par un détective (cf. Gaatone 1998, 194-210). Dans cette optique, le SPrép en de après un verbe de changement chromatique présenterait donc la couleur (ou encore la substance colorante) comme la cause du changement de couleur, une cause peu dynamique (parce que de nature inanimée) plutôt que comme le résultat du processus, qui, lui, pourrait en être déduit par inférence (un $X$ coloré de jaune $\rightarrow X$ est jaune). L'exemple suivant souligne en outre la proximité avec les compléments de cause construits par la préposition de (p. ex. briller de gaieté, frémir de peur). 
(46) Tant de chaleur ne peut rester sans effet. Le visage de Charles se colore d'une rougeur, et ses yeux brillent de gaieté. (Tournier M., Gilles et Jeanne, 1983, 12)

Toutefois, plusieurs observations relatives aux emplois pronominaux de colorer (peindre n'est pas attesté en emploi pronominal) semblent infirmer cette hypothèse. D'une part, les emplois neutres de se colorer (en tout 22 exemples avec de) sont caractérisés par l'absence d'un facteur causal externe. C'est d'ailleurs ce qui distingue un emploi neutre d'un emploi passif (Melis 1990, 112-114). Ce dernier suppose un agent (le plus souvent humain), qui reste cependant implicite. Comparez :

(47) a. Les œufs se cuisent à feu doux. (passif, agent implicite)

b. Le sel se cristallise. (neutre, sans agent)

En emploi neutre, on ne s'attend donc pas à la présence d'un complément d'agent, fûtil affaibli. Par conséquent, il ne semble pas que le complément en de du verbe colorer puisse être un complément d'agent. Certes, certains verbes neutres - notamment ceux qui véhiculent un état d'âme - tendent à se construire avec un complément de cause affaibli, qui exprime le facteur qui a provoqué l'état d'âme. Ce complément correspond de ce fait au sujet-thème de la construction active :

(48) a. Il se réjouit de son succès. (cf. aussi étonner, alarmer, préoccuper, réjouir, ...)

b. $\sim$ Son succès le réjouit.

Toutefois, la couleur (à la différence des substances colorantes ou d'autres sources de la coloration, telles que le coucher $d u$ soleil, Bernez 2014, 180) passe difficilement à la position sujet, ce qui montre que l'analogie n'est pas justifiée :

(49) a. Quand nous levons les yeux, nous voyons s'agrandir un ciel d'une pâleur limpide qui déjà se colore de bleu. (Genevoix M., Ceux de 14, 1950, 668)

b. ${ }^{*}$ Du bleu colore le ciel.

D'autre part, l'emploi pronominal à valeur passive n'autorise pas non plus l'expression explicite d'un complément d'agent, sauf circonstances exceptionnelles (cf. supra). Là encore, on ne s'attend pas non plus à l'apparition d'un complément d'agent, attente contredite par l'exemple suivant, le seul exemple pronominal passif du corpus :

(50) initialement blanc avec des dessins rouges, il s'était coloré de rose pisseux à force de lavages

(Manchette J. P., Trois hommes à abattre : le petit bleu de la côte ouest, 1976, 101)

On peut en déduire que ce complément en de n'est pas un complément d'agent. On notera que la source (externe) du changement de couleur est cependant présente dans le contexte, sous la forme d'un complément circonstanciel de cause (à force de lavages), ce qui est bel et bien possible pour un passif pronominal. La juxtaposition d'un complément circonstanciel de cause constitue d'ailleurs une preuve supplémentaire du fait que le complément en de n'est pas un complément d'agent auprès d'un prédicat passif. De même, dans l'exemple neutre (51), proche d'un emploi passif, la cause de l'effet de coloration (à la lumière) apparaît dans le contexte, en plus du complément en de : 
(51) Transparentes, elles se coloraient, à la lumière, de rose et d'incarnat. (France A., Le Petit Pierre, 1918, 257)

Le coup de grâce est donné par l'exemple suivant, qui combine un complément en de avec un complément d'agent en par :

(52) Le coteau sur lequel le vignoble est placé est un sol argilo-calcaire, coloré de rouge par des oxydes de fer. (Huysmans J. K., L'Oblat, 1903, 113)

En somme, si les compléments en de semblent fortement liés à la diathèse décausative, on ne saurait les considérer comme des compléments d'agent de prédicats passifs. Le changement de couleur y est plutôt un procès auto-causé (= neutre, donc ni actif, ni passif) ou causé par un agent / une cause externe qui reste le plus souvent implicite. On peut cependant soupçonner que de garde une trace d'une nuance causale (cf. rouge de colère, bleu de rage) ou instrumentale, qui n'est pas présente dans le sémantisme de en. L'analogie est palpable avec des verbes comme habiller, qui se combinent d'ailleurs aussi avec des compléments comportant des noms de couleur :

(53) habillé en bleu / de bleu

(54) Je m'habille en jeans / d'un jeans. ${ }^{24}$

5.3 Affectation exhaustive et exclusive du support

La dernière variable qui régit l'alternance en/de est plus subtile, car elle est liée à la sémantique des prépositions : en aide à construire des changements chromatiques qui affectent le support de manière exhaustive, le caractérisant de manière holistique et exclusive, ce qui revient à dire que le résultat sera de préférence unicolore. $D e$, en revanche, accepte facilement des résultats multicolores dans la mesure où la prédication chromatique n'affecte pas le référent de manière exhaustive et exclusive. Cette thèse sera étayée au moyen de deux types d'arguments distributionnels : les régimes pluriels (section 5.3.1) et les régimes coordonnés (section 5.3.2).

\subsubsection{La pluralité des $N$ régime}

Peindre et colorer construits avec la préposition de se combinent 12 fois avec un nom au pluriel ( $8 / 28$ pour peindre et $4 / 55$ pour colorer), sur un total de 83 occurrences, soit $14,46 \%$. Avec en, en revanche, ce pourcentage chute à 1,96\%, soit 5 exemples au pluriel sur 255 (4/142 pour peindre en, $1 / 113$ pour colorer en $)\left(\mathrm{X}^{2}=17.941, \mathrm{df}=1, \mathrm{p}<0.001\right)$. Notons que la plupart $(15 / 17)$ de ces occurrences comportant un nom au pluriel concernent

24 Les locuteurs sont conscients de la concurrence entre les deux : <https://forum.wordreference.com/ threads/shabiller-de-ou-shabiller-en.1575082/>, dernier accès : 25/06/2018. 
des noms comme couleurs ou dans une moindre mesure nuances (en couleurs vives, avec des couleurs plus pimpantes, ...), stratégie de pluralisation plus naturelle que la pluralisation pure et simple d'un nom de couleur. En effet, celle-ci est plus limitée sur le plan de la variation chromatique (il s'agit toujours de nuances d'une seule couleur) et demande en outre une conversion taxonomique du massif au comptable (p. ex. en bleus de diverses tonalités $=$ 'sortes de bleu').

Ce lien privilégié entre de et la polychromie est confirmé par une rapide recherche dans Frantext catégorisé (ensemble du corpus, 1830-1997) :

de : \&cpeindre de couleurs : 52 ; \&ccolorer de couleurs : 1

en : \&cpeindre en couleurs : 12 ; \&ccolorer en couleurs : 0

Le contraste entre de et en n'est par ailleurs pas à mettre sur le compte des contraintes structurelles imposées par en, qui n'accepte guère les SN complets (cf. section 5.1), car le même biais pour le pluriel s'observe dans notre corpus si on s'en tient aux seuls N' (noms nus, accompagnés d'un modificateur) : de (11) vs en (3). En outre, ce n'est pas tant le nom couleur qui provoque une certaine aversion du côté de la préposition en, puisque $\left[e n+\right.$ couleur $\left._{\text {singulier }}\right]$ est même plus fréquent que $\left[d e+\right.$ couleur $\left._{\text {singulier }}\right]: 4$ attestations contre une seule. Dans l'ensemble du corpus Frantext catégorisé (1830-1997), peindre (conjugué) en couleur est aussi fréquent que peindre (conjugué) de couleur (8 attestations des deux côtés). Il ressort de ce qui précède que la surreprésentation des noms au pluriel avec de par rapport à en semble donc liée aux propriétés des constructions prépositionnelles. C'est ce que nous allons regarder de plus près.

Rappelons tout d'abord que le singulier reste l'option par défaut ( $>85 \%$, même pour de) : d'habitude un changement chromatique aboutit à un nouvel état monochromatique. La préposition en semble ériger cette tendance presque en contrainte sémantique, alors que de est moins restrictif. De ce fait, de s'aligne sur les autres prépositions $(\grave{a}$, avec, par) dont le caractère instrumental se marie facilement avec une pluralité de couleurs-instruments : 10 des 23 occurrences présentent en effet un régime au pluriel, soit $43,5 \%$.

Le quasi-rejet de la pluralité après en peut être mis en rapport avec le comportement de celui-ci dans d'autres contextes. En effet, en, notamment dans le domaine spatial-fonctionnel, est en général suivi d'un nom au singulier, sans déterminant : en ville, en ligne, en prison, en face, en mai, en vélo, en costume, ... Tous ces emplois présentent le même déficit catégoriel provoqué par l'absence de déterminant, comme le montre la perte de la modification adjectivale :

(55) (peint) *en jaune très original $\rightarrow$ dans/en un jaune très original

(56) Pierre est* en prison rénovée.

(57) * Jean est habillé en jaune très original.

Ce déficit catégoriel avec les termes de couleur s'observe donc indépendamment du verbe, comme le montre (57). En somme, l'absence du pluriel après en ne s'avère donc pas une contrainte propre aux verbes de changement chromatique, mais il n'en reste pas moins que, appliquée aux verbes chromatiques, elle a des conséquences assez impor- 
tantes, dans la mesure où ceux-ci n'arrivent pas à exprimer un état chromatique multicolore exprimé par un pluriel.

\subsubsection{Les SPrép résultatifs coordonnés}

Toutefois, on ne saurait réduire la contrainte 'monochrome' de en à une simple contrainte morphosyntaxique plus générale qui déterminerait la forme de son régime (nom sans déterminant, au singulier, cf. section 5.3.1). La sémantique de la préposition joue également un rôle. C'est ce que montre l'examen de la coordination de SX résultatifs.

En effet, les deux prépositions en et de ne se comportent pas de la même manière lorsqu'il s'agit d'exprimer un résultat multicolore au moyen de deux SPrép coordonnés (comportant des $\mathrm{SN}$ au singulier) :

(58) Mais sur la plage, un poteau peint en rouge et blanc indiquait l'emplacement d'un téléphone de campagne. (Littell J., Les Bienveillantes, 2006, 863 sq.)

(59) dans un petit appartement aux murs peints en rose vif et en violet (Aubry G., Personne, 2009, 70 sq.)

C'est le cas de 36 exemples du corpus (sur un total de $338 \mathrm{en} / \mathrm{de}$ ). Là encore, de se combine plus souvent que en avec des régimes coordonnés : $13,25 \%(11 / 83)$ contre $9,8 \%$ $(25 / 255)$, mais cette différence n'est pas significative $\left(X^{2}=0.46229, \mathrm{df}=1, \mathrm{p}>0.05\right)$. Il faut d'ailleurs encore nuancer ces résultats, car il y a coordination et coordination. Un premier type de coordination, [en $\left(\mathrm{N}_{1} \mathrm{Cco} \mathrm{N}_{2}\right)$ ], se situe à l'intérieur du régime et coordonne deux noms, comme en (58). Comme en/de sont des prépositions qui en principe doivent être répétées (cf. Il a été en Chine et en Russie vs *en Chine et Russie), on ne peut pas y voir le résultat d'une ellipse. Si la deuxième préposition fait défaut, c'est qu'il n'y en a pas, tout simplement. Il s'ensuit qu'on a affaire à des noms coordonnés dépendant de la même tête prépositionnelle. Sur le plan sémantique, le résultat est tout naturellement bicolore, les deux couleurs étant réunies dans une seule opération de changement chromatique.

Or, on peut également envisager la coordination d'une autre façon, entre deux SPrép - donc [(de N1) Cco (de N2)], Cco étant la conjonction de coordination -, comme en $(60)$, ce qui donne lieu à une double opération de changement chromatique :

(60) On nous a fait asseoir dans un couloir peint de beige sale et de vert chlorophylle. (Aubry G., Personne, 2009, 138 sq.)

Les deux prépositions ne se comportent pas de la même manière face à ces deux types de coordination. Avec de, la préposition est répétée dans 10 coordinations sur 11, alors que pour en il n'y a reprise de la préposition que dans la moitié des cas (12/26). Parmi ces 12 exemples, on en trouve 3 qui répartissent le changement chromatique sur deux surfaces complémentaires, qui ne se recoupent pas : 
(61) Les murs de la salle d'étude étaient peints en gris à mi-hauteur et en bleu ciel jusqu'au plafond. (Levy M., L'étrange voyage de Monsieur Daldry, 2011, 315)

En outre, tous les exemples avec en se construisent avec un support pluriel ou massif, qui autorise une lecture distributive :

(62) Merveilleux plafonds, profonds, creusés, peints en vert, en bleu et or. (Fellous C., Avenue de France, 2001, 1445)

En clair, le résultat peut être à chaque fois unicolore (lecture distributive), chaque item au sein de la pluralité des objets ayant sa propre couleur, ce qui permet de contourner la contrainte de l'unicité de la couleur. La lecture distributive est souvent renforcée par une disjonction logique (ou) ou chronologique (d'abord en $X$, puis en $Y$ ), qui chacune à leur manière 'sauvent' l'unicité du changement chromatique :

(63) des baraques [pluriel distributif] peintes en orange ou en rose (Weyergans F., La Vie d'un bébé, 1986, 109 sq.)

En somme, pour en, il ne reste aucun exemple dans lequel un support de nature comptable, au singulier, fait l'objet d'un processus de coloration qui aboutit à un état polychrome. Il n'en est pas de même de la préposition de :

(64) une carte du Maghreb, colorée de vert amande, d'ocre et de rose (Simon C., Les Géorgiques, 1981, 111)

En tout, le corpus contient 4 attestations de ce type, où tout soupçon de lecture distributive est absent.

Le nombre d'exemples étant extrêmement bas, nous avons procédé à un petit sondage supplémentaire dans un corpus plus grand, le corpus frTenTen, un corpus web fermé de 10 milliards de mots (Kilgarriff et al. 2014). Cette vérification nous permet aussi d'écarter l'impact de la structure morphosyntaxique des SX chromatiques. En effet, dans la moitié des exemples $(11 / 23)$ avec répétition de la préposition (tant en que de), on note la présence de modificateurs sur le premier membre de la coordination (ou d'autres éléments qui rompent la cohésion à l'intérieur du syntagme coordonné), ce qui exclut presque la possibilité de ne pas répéter la préposition (cf. ex. (60) et (64)). Pour exclure ce facteur, nous avons donc limité la comparaison entre en et de à des coordinations sans modification du premier membre. Voici les résultats pour les coordinations comportant bleu :

(65) $E N$

en bleu : $\quad[$ lemma $=\ll$ peindre $»][$ word $=\langle$ en $\gg][$ word $=\langle$ bleu $\gg]: 1573$

en bleu et en $\mathrm{Y}:[$ lemma $=\langle$ peindre $»][$ word $=\langle$ en $\gg][$ word $=\langle$ bleu $\gg][$ word $=\langle$ et $\gg]$ $[$ word $=\langle$ en $\gg]: 16$

(66) $D E$

de bleu : $\quad[$ lemma $=\langle$ peindre $\gg][$ word $=\langle$ de $\gg][$ word $=\langle$ bleu $\gg]: 55$

de bleu et de $\mathrm{Y}:[$ lemma $=\langle$ peindre $\gg][$ word $=\langle$ de $\gg][$ word $=\langle$ bleu $\gg][$ word $=\langle$ et $\gg]$

$[$ word $=\langle$ de $\gg]: 6$ 
On constate que 6 exemples sur 55 comportent une coordination de deux SPrép en de, soit 10,9\%. Pour en, le rapport est seulement de 16 à 1573, soit 1,0 \% $\left(\mathrm{X}^{2}=31.939, \mathrm{df}=1\right.$, $\mathrm{p}<0.001$ ). Le même contraste entre en et $d e$ s'observe d'ailleurs avec d'autres verbes tels que habiller (corpus frTenTen), en combinaison avec des termes de couleur (en l'occurrence bleu et jaune) :

(67) bleu : 9 occ. de habillé de bleu et de Y sur un total de 126 occ. de habillé de bleu /vs/ o occ. de habillé en bleu et en $Y$ sur un total de 146 occ. de habillé en bleu

(68) jaune : 3 occ. de habillé de jaune et de $Y$ sur un total de 38 occ. de habillé de jaune /vs/ 1 occ. de habillé en jaune et en $Y$ sur 59 occ. de habillé en jaune

En définitive, il semble donc qu'avec en, quel que soit le prédicat verbal dont il dépend, les locuteurs évitent de coordonner deux résultats chromatiques simultanés. De est moins contraint sur ce point. Comment expliquer ce contraste ? Il convient de rappeler qu'il n'a rien à voir avec les contraintes liées à la répétition obligatoire de la préposition, car en principe les deux prépositions doivent être répétées. La différence semble plutôt liée à la conceptualisation de la relation véhiculée par la préposition en, et confirme les observations faites ci-dessus pour les noms pluriels (section 5.3.1). Pour éclairer notre lanterne, il convient de revenir sur le verbe habiller. Il s'avère que le contraste entre en et de se reproduit avec d'autres types de noms :

(69) a. Habillé d'un jeans et d'un pull-over

b. ?Habillé en jeans et en pull-over

C'est finalement l'analyse de Leeman qui va nous fournir la clé du mystère : dans (69b) en introduit un 'vêtement qui permet de distinguer la personne par sa tenue' (Leeman 1996, 82 sq.). Du coup, être en jeans / en pull-over / en costume caractérise la personne de manière globale, l'effet caractérisant étant facilité sans doute par l'absence de déterminant qui annule l'individuation du référent vestimentaire. Si on accepte ces prémisses, il se révèle plus difficile de superposer deux caractérisations globales qui affectent toutes les deux la personne de manière 'holistique'. Par contre, avec de, l'impact de la caractérisation est plus 'local' et partant non exclusif, ce qui rend la juxtaposition (et donc superposition) de deux caractérisations plus naturelle. $D e$ ne fait qu'introduire un vêtement construit en 'instrument/moyen' (section 5.2.2), qui n'est pas nécessairement exclusif : rien ne nous empêche de porter plusieurs vêtements à la fois. De même, un support peut être affecté en même temps par trois 'instruments chromatiques' différents, qui agissent en parallèle. Ceux-ci affectent le référent de manière non exhaustive à travers un processus que l'on pourrait associer à un processus de remplissage ou de recouvrement chromatique : peint de vert $\sim$ couvert d'une couche de peinture verte.

Quant à l'origine de cette tendance à l'exclusivité qui caractérise $e n$, on pourrait émettre l'hypothèse selon laquelle le changement de couleur serait fondamentalement basé sur l'image schema (Johnson 1987) du déplacement spatial vers un container : Pierre entre \{dans la maison/en ville\}. Pour un référent sujet non pluriel, il est difficile d'entrer dans plusieurs relations d'inclusion spatiale en même temps : ?Sur le coup de 8 heures, Pierre entre dans la petite maison et dans la grande maison; ?Sur le coup de 8 heures, Pierre 
entre dans trois maisons. Ces énoncés ne sont interprétables qu'en lecture disjonctive successive (l'une après l'autre). De même, il est difficile de faire entrer une porte en trois états chromatiques différents au même moment (?Pierre peint la porte en rouge, en bleu et en vert). Cependant, l'aspect non global du présent permet facilement d'inclure cet événement multiple (comportant 3 phases successives) dans la sphère du présent, ce qui rend ces phrases tout à fait acceptables pour les 4 locuteurs natifs que nous avons interrogés. L'image schema évoquée par de serait différent : la porte se couvre / se remplit en quelque sorte de trois couleurs, c'est-à-dire la porte subit l'action de trois instruments qui peuvent agir parallèlement. Il s'agirait donc plutôt d'un schéma de superposition spatiale (recouvrement) (cf. covering, Lakoff 1987) qui ne ressemble en rien à l'entrée dans un espace tricolore.

En somme, même s'il ne s'agit que de tendances qui renforcent la tendance 'unicolore' dominante - qui caractérise à la fois en et de -, nous pensons tout de même avoir mis le doigt sur certaines différences subtiles dans la conceptualisation des deux prépositions. Là où en encode un changement chromatique, conçu comme l'entrée dans un état, qui affecte de manière exhaustive et exclusive le support (qui s'en trouve caractérisé), de est compatible avec une série d'états chromatiques simultanés qui affectent le référent de manière non exhaustive à travers un processus de recouvrement chromatique. Ces différences sont en partie indépendantes des contraintes syntaxiques imposées par en à son régime et s'observent aussi dans d'autres contextes, notamment avec des verbes comme habiller.

\section{Conclusions}

Si nous revenons maintenant sur les questions que nous nous sommes posées au début, nous espérons avoir montré que :

1. Le français tolère marginalement les SAdj résultatifs si le contexte atténue 'le choc' causé par l'adjonction d'un adjectif, en mettant en place une configuration plus proche de la configuration dépictive par le biais de l'aspect grammatical résultatif du verbe et de la syntaxe appositive.

2. Si le SPrép reste l'option par défaut en français, force est de constater que la gamme des prépositions est beaucoup plus vaste : en (et sa variante combinatoire dans), de, $\grave{a}$, par et avec. Certes, ces trois dernières construisent des compléments d'instrument qui n'expriment qu'indirectement le changement de couleur, tirant profit d'une certaine sous-détermination sémantique des noms de couleurs. En revanche, avec en et $d e$, on se rapproche des constructions résultatives à proprement parler.

3. Le choix entre de et en n'est pas aléatoire. Il est en premier lieu orienté par les propriétés diathétiques du prédicat (et dans une moindre mesure par celles du verbe, e. a. les sujets inanimés), de étant lié à une visée décausative ou déagentive (passive ou neutre, périphrastique ou pronominale), qui, pour colorer, peut également être obtenue par un sujet inanimé. En, de son côté, n'est pas vraiment sélectif (même si, pour peindre, le biais actif est net). Si le complément en de n'est pas à considé- 
rer comme un complément d'agent, force est de constater qu'il se rapproche d'un complément d'instrument (ou de cause) construisant la couleur comme un principe actif recouvrant le support d'une substance colorante. Un deuxième facteur qui semble régir la distribution de en et de concerne l'affectation 'holistique' et donc exclusive induite par la localisation abstraite réalisée par la préposition en, qui rend plus difficile l'attribution d'états multicolores. Cette contrainte est encore renforcée par les restrictions catégorielles imposées par en à son régime. En ne tolère pas des $\mathrm{SN}$ complets, contrainte étrangère à de, qui, lui, se combine volontiers avec des $\mathrm{SN}$ complexes, dénotant parfois des couleurs originales et subjectives.

Maintenant que le lecteur aura son siège fait, il importe de revenir sur la question de fond : toutes ces constructions sont-elles des réalisations de la construction résultative et le sont-elles au même titre?

$\mathrm{Si}$ les variantes instrumentales ont tout au plus un lien indirect avec la construction résultative, la question paraît plus délicate pour de et en. Etant donné que la préposition de semble conserver une trace de sa sémantique causale/instrumentale - sans que pour autant on puisse parler d'un complément d'agent -, elle se rapproche de la triade avec/ par/à, avec laquelle elle partage l'affectation locale et donc non exclusive du support. Cette nuance se marie très bien avec le schéma cognitif du recouvrement (peint de / couvert de) qui profile encore davantage la source du changement chromatique, alors que en indique uniquement un changement d'état, métaphore du changement de lieu. De ce fait, la structure argumentale (au sens large, impliquant aussi des compléments non nucléaires) impliquée par en est plus proche de la configuration sémantique prototypique de la construction résultative. Ce rapprochement se manifeste aussi au niveau morphosyntaxique. En effet, en se rapproche à certains égards de la construction adjectivale, et cela à la fois par son propre statut catégoriel et par le statut catégoriel de son régime. Comme la construction [en $+\mathrm{N}$ de couleur] peut être rattachée au spectre de l'attribut (de l'objet), plutôt qu’à celui des compléments de manière (Dagnac 2009), contrairement à ce que pensent Grevisse/Goosse (2011,438), il n'est pas surprenant que la préposition perde certaines caractéristiques de sa catégorie pour devenir une sorte de marqueur prédicatif (Lauwers/Tobback 2018). Cela ressort notamment du fait qu'elle se combine avec des adjectifs dans une construction comparative (et l'accord en fait foi) :

(70) Lise, c'est Rose en plus maline / en moins blonde / ??en grande. (Noailly 2010, 199)

Comme le montre Dagnac (2009, 77), en se basant sur les arguments de Noailly (2010), ${ }^{25}$ cette analyse adjectivale se défend même lorsque en fait suite à un verbe de changement de couleur :

(71) Teindre les rideaux en plus rouge que la dernière fois. 
Que dans ces cas on puisse bel et bien avoir affaire à des régimes adjectivaux peut être déduit du fait que certains régimes n'ont pas de contrepartie nominale (clair, vif, sombre) :

(72) Il faut que je teigne mes rideaux en (plus) sombre.

(73) ?? Le sombre lui va bien.

(74) ?? Il faut que je teigne mes rideaux dans un sombre plus accentué.

En outre, ces termes peuvent être précédés d'adverbes de degré, qui pointent eux aussi vers le domaine adjectival (en très clair, en plus vif, etc.). Dagnac $(2009,78)$ va jusqu’à accepter une toute petite ouverture vers l'accord en nombre (il a repeint ses volets en plus sombres).

Certes, cette analyse adjectivale ne se défend pas dans tous les cas :

(75) Le plafond est peint en (*plus) couleur brique.

En outre, le fait que $[e n+\mathrm{N}]$ puisse être coordonné à $[$ dans + dét + N] (Dagnac 2009, 77 ), construction dans laquelle $\mathrm{N}$ est clairement nominal, montre que l'analyse nominale ne doit pas être abandonnée dans tous les cas :

(76) Le socle quant à lui est peint en rouge et dans diverses nuances de vert. ${ }^{26}$

Il n'entre pas dans notre propos d'apporter la solution définitive à cet épineux problème, mais ce qu'il faut retenir ici, c'est que le SPrép [en $+\mathrm{N}$ de couleur] est peut-être plus proche d'un SAdj qu'on ne le pense. Même le contre-argument le plus décisif, à savoir l'absence de l'implication attributive $(\hat{e} t r e+X)$, n'est peut-être pas aussi percutant qu'on ne le croit :

(77) a. The door is painted red. $\rightarrow$ The door is red.

b. La porte est peinte en rouge. $\rightarrow{ }^{*}$ La porte est en rouge. (Dagnac 2009, 76)

En effet, l'implication être en $X$ ne semble pas tout à fait exclue lorsque la couleur est perçue comme une propriété passagère, circonstancielle, conformément au sémantisme de base de en, dénotant des propriétés non inhérentes (Amiot/De Mulder 2013, 28), comme le rappelle Dagnac elle-même :

(78) Dans cette reproduction les volets sont en vert. (Dagnac 2009, 77)

(79) Bonjour, je viens de mettre ma voiture en vente et le prix de vente est en rouge dans ma rubrique 'objet en vente'. Est-ce normal. $[\text { sic }]^{27}$

Avec de (et les autres prépositions), en revanche, une telle implication est exclue :

(8o) ${ }^{*}$ La porte est de rouge.

26 <https://www.museedujouet.eu/index.php/jouet/touslesjouets/fiche?idj=776>, dernier accès : 25/o6/ 2018 .

27 <https://communaute.ebay.fr/t5/vendre-sur-ebay/le-prix-de-mon-objet-de-vente-est-en-rouge/ qaq-p/404951>, dernier accès : 25/06/2018. 
En somme, notre examen nous amène à proposer l'échelle suivante, le SX adjectival étant la réalisation prototypique de la construction résultative : adjectif $<e n<d e<\dot{a}$ / par/avec. Cette échelle peut faire office de carte sémantique des constructions marquant de manière analytique le changement chromatique. Reste à tester son utilité pour d'autres langues. Pour l'instant, nous nous limitons à signaler que le français semble se rapprocher un peu plus de la configuration prototypique, étant donné que même les SAdj sont attestés dans certains contextes bien précis.

\section{Références}

Amiot, Dany / De Mulder, Walter (2013) : «En : de la préposition à la construction », in : Langue française 178, p. 21-39.

Bernez, Cécilia (2014) : Grammaire des couleurs, Francfort-sur-le-Main : Peter Lang.

Boas, Hans C. (2003) : A Constructional Approach to Resultatives, Stanford : CSLI.

Boas, Hans C. (2011) : « Coercion and leaking argument structures in Construction Grammar », in : Linguistics 49, p. 1271-1303.

Burnett, Heather / Troberg, Michelle (2014): « On the diachronic semantics of resultative constructions in French $\gg$, in : Empirical Issues in Syntax and Semantics 10, p. 37-52.

Cadiot, Pierre (1991) : «À la hache ou avec la hache ? Représentation mentale, expérience située et donation du référent $\gg$, in : Langue française 91, p. 7-23.

Dagnac, Anne (2009) : «Elle a teint ses rideaux en rouge : entre manière et résultativité », in : Langages 175, p. $67-84$.

Dowty, David (1991) : « Thematic proto-roles and argument selection », in : Language 67, p. 546619.

Farkas, Imola-Ágnes (2009) : «Some differences between English and Romanian resultative constructions $\gg$, in : Bucharest Working Papers in Linguistics 2, p. 59-71.

Farkas, Imola-Ágnes (2011) : « Resultative expressions in Romanian », in : Ianua 11, p. 67-88.

Gaatone, David (1998) : Le passif en français, Paris/Louvain-la-Neuve : Duculot.

Grevisse, Maurice/Goosse, André (2011) : Le Bon Usage. Grammaire française, Bruxelles : de Boeck/Duculot.

Himmelmann, Nikolaus P. / Schultze-Berndt, Eva F. (2005) : « Issues in the syntax and semantics of participant-oriented adjuncts : an introduction $\gg$, in : idem (éds.), Secondary Predication and Adverbial Modification: The Typology of Depictives, Oxford : Oxford UP, p. 1-67.

Johnson, Mark (1987) : The Body in the Mind : The Bodily Basis of Meaning, Imagination, and Reason, Chicago/Londres : Univ. of Chicago Press.

Kilgarriff, Adam / Baisa, Vít / Bušta, Jan et al. (2014) : « The sketch engine : ten years on », in : Lexicography 1, p. 7-36.

Kleiber, Georges (2011) : « Types de noms : la question des occurrences », in : Cahiers de lexicologie 99, p. 49-69.

Lakoff, George (1987): Women, Fire, and Dangerous Things: What Categories Reveal about the Mind, Chicago/Londres : Univ. of Chicago Press.

Lauwers, Peter/Willems, Dominique (2011): « Coercion : Definition and challenges, current approaches, and new trends $\gg$, in : Linguistics 49, p. 1219-1235.

Lauwers, Peter / Tobback, Els (2018) : «La contribution du paradigme comme, pour, de et en au marquage de la relation attributive $\gg$, in : Quand les formes prennent sens - grammaire, prépositions, constructions, système, éd. par C. Vaguer-Fekete, Limoges : Lambert-Lucas, p. 249-260. 
Leeman, Danielle (1996) : «Vêture, coiffure, chaussures et autres coquetteries », in : Sémiotique, phénoménologie, discours. Du corps présent au sujet énonçant, éd. par M. Costantini et I. Darrault-Harris, Paris : L’Harmattan, p. 79-89.

Legendre, Géraldine (1989) : «Unaccusativity in French », in : Lingua 79, p. 95-164.

Legendre, Géraldine (1997) : « Secondary predication and functional projections in French », in : Natural Language and Linguistic Theory 15, p. 43-87.

Martínez Vázquez, Montserrat (1998) : «Effected objects in English and Spanish », in : Languages in Contrast 1, p. 245-264.

McLaughlin, Mairi (2010) : «L'influence de l'anglais sur la syntaxe du français : une étude de cas concernant la voix passive », in : Actes du Congrès Mondial de Linguistique Française - CMLF 2010, éd. par F. Neveu, V. Muni Toke, J. Durand et al., <https://www.linguistiquefrancaise.org/ articles/cmlf/pdf/2010/01/cmlf2010_000221.pdf >, dernier accès : 11/12/2017.

Melis, Ludo (1990) : La voie pronominale. $\bar{L}$ a systématique des tours pronominaux en français moderne, Paris/Louvain-la-Neuve : Duculot.

Muller, Claude (2001) : «Classes de verbes français transitifs à extension attributive : les opérateurs supplétifs », in : Langage et référence. Mélanges offerts à Kerstin Jonasson à l'occasion de ses soixante ans, éd. par H. Kronning, C. Norén, B. Novén et G. Ransbo, Upsal : Uppsala University Library, p. 419-429.

Napoli, Donna Jo (1992) : «Secondary resultative predicates in Italian », in : Journal of Linguistics 28 , p. 53-9o.

Noailly, Michèle (2010) : «L'adjectif, quand il est invariable, sort-il pour autant de sa catégorie ? », in : L'adjectif hors de sa catégorie. Actes du VI colloque de linguistique franco-roumaine. Université d'Artois, 23-25 mai 2007, éd. par J. Goes et E. Moline, Arras : Artois Presses Université, p. 189202.

Rodríguez Arrizabalaga, Beatriz (2002) : Attribution in Contemporary English and Spanish: Contrasts in Expressing Change of State/La atribución en inglés y español contemporáneos. Contrastes en la expresión del cambio de estado, PhD, University of Michigan, Ann Arbor : UMI Dissertation Services.

Rodríguez Arrizabalaga, Beatriz (2016) : « Construcciones resultativas en español. Caracterización sintáctico-semántica $\gg$, in : Philologica Canariensia 22, p. 55-87.

Slobin, Dan I. (2004) : « The many ways to search for a frog : Linguistic typology and the expression of motion events $\gg$, in : Relating Events in Narrative. Vol. 2 : Typological and Contextual Perspectives, éd. par S. Strömqvist et L. Verhoven, Mahwah/Londres : Lawrence Erlbaum Associates, p. 219-257.

Talmy, Leonard (1991) : « Path to realization : A typology of event integration », in : Buffalo Papers in Linguistics 1, p. 147-187.

Talmy, Leonard (2000) : Toward a Cognitive Semantics. Vol. 1 : Concept Structuring Systems, Londres/ Cambridge, Massachusetts : MIT Press.

Togeby, Knud (1982) : Grammaire française. Vol. 1 : Le nom, éd. par M. Berg et G. Merad, Copenhague : Akademisk Forlag.

Vénérin-Guénez, Christine (2013) : «Attribut du complément ou complément de manière ? En morceaux : quel casse-tête! », in : Scolia 27, p. 141-160.

Wechsler, Stephen (2001) : «An analysis of English resultatives under the event-argument homomorphism model of telicity », in : Proceedings of the 3 rd Workshop on Text Structure, University of Texas, Austin, Oct. 13-15, 2000, <http://citeseerx.ist.psu.edu/viewdoc/download?doi=10.1.1.9. 958\&rep=rep1\&type $=$ pdf $>$, dernier accès $: 11 / 12 / 2017$. 


\section{Corpus}

Frantext $=$ Base textuelle Frantext, ATILF-CNRS et Université de Lorraine, <http://www.frantext. fr >, dernier accès : 10/12/2017.

frTenTen = French corpus from the web, Sketch Engine, Sussex/Brno : Lexical Computing, <http:// www.sketchengine.eu/frtenten-french-corpus $>$, dernier accès : 10/12/2017.

PETER LAUWERS, RENATA ENGHELS \& MACHTELD DUFOUR

Université de Gand, Département de Linguistique, Blandijnberg 2, 9000 Gand, Belgique, Peter.lauwers@ugent.be, Renata.enghels@ugent.be, machtelddufour@hotmail.com 